\title{
GEOMETRIC RELIABILITY MODEL OF THE FIVE SITE REDUNDANT STRUCTURE
}

\author{
Valery Usenko $^{{ }^{*}}$ - Olga Kodak ${ }^{2}$ - Iryna Usenko ${ }^{3}$
}

\begin{abstract}
${ }^{1}$ The Department of Descriptive Geometry and Graphics, Arhitectural Faculty, Poltava National Technical Yuri Kondratyuk University, 24, Pershotravnevyi Avenue, Poltava, Ukraine

${ }^{2}$ The Department of Descriptive Geometry and Graphics, Arhitectural Faculty, Poltava National Technical Yuri Kondratyuk University, 24, Pershotravnevyi Avenue, Poltava, Ukraine

${ }^{3}$ The Department of Hidraulics, Water Supply and Sewerage, Faculty of Civil Engineering, Poltava National Technical Yuri Kondratyuk University, 24, Pershotravnevyi Avenue, Poltava, Ukraine
\end{abstract}

\begin{tabular}{l} 
ARTICLE INFO \\
\hline Article history: \\
Received: 2.1 . 2018. \\
Received in revised form: 29. 92018. \\
Accepted: 5. 10. 2018. \\
\hline Keywords: \\
Geometric modeling \\
Structural modeling \\
Connectivity probability \\
\hline DOI: http://doi.org/10.30765/er.40.2.02
\end{tabular}

\section{Introduction}

In the issue of ensuring the reliability of systems there is a need for an integrated approach in a number of scientific areas. The integrated task solution increases their efficiency and helps to obtain qualitatively new results.

Reliability of existing water supply systems is constantly decreasing. The postponement of necessary maintenance and replacement of the system components may lead to disaster [1]. Water supply networks are complex systems that require optimization [2]. Probability of connectivity is also an important issue in the design and reconstruction

\begin{abstract}
:
The study results from the graphical representation on connectivity probability of systems that have structural redundancy for modeling. The structural reliability of different systems is presented. A oneparameter expression of a two-ring structure reliability with five sites is described. All the unique operating conditions of the structure are shown in a graphical form. The compact form of the exact expression of the redundant two-ring structure reliability with five sites is presented, which is convenient for computer modeling. The peculiarities of the dependences of many variables in the reliability model of the two-ring structure with five sites and four nodes are determined. It is shown that the graphic representation of the dependence of the five variables helps to study the properties of multiparameter dependencies. The components of a geometric model of various dimensions are considered in detail.
\end{abstract}

of other engineering networks: gas, electric, networks of cellular operators, etc. [3].

Risks in the supply chain management cause negative consequences in the system of objects that are part of the distribution network. [4]. The failure rate growth in supply chain is affected by the nature of the violation, structure and dependence, as well as the managerial decision-making. The higher level of failure detalization reveals the following factors of reliability of the system operation: risk ratio, compaction effects, cyclic relationships and counterparty risk [5].

Due to the high level of demonstrativeness, computer graphic modeling of various dependencies is widely used in the fields of science and

\footnotetext{
* Corresponding author.

E-mail address: valery.usenko@ukr.net
} 
production [6]. In the process of solving many theoretical and practical problems, special images of multidimensional geometric shapes are used. The problem of constructing effective methods of computer graphic modeling of objects of multidimensional space remains important and relevant. In the mathematical modeling for reliability there is a need to accurately determine the connectivity probability of system structures with different reliability of elements. This article considers the reliability of systems with the structure described by the graph of the network [3]. The object of research has a higher level of generalization than a communication network or a transport network. The examples of principles representing the structures of the complex systems and the processes of fulfilling the tasks by the renewable systems are given. Limit values of reliability indicators are presented for the complex systems. The publication [7] highlights the basis of the theory of structurally-complex systems reliability. The analysis of the complex systems relies on the basis of the probabilistic logic theory. For reliability calculations the problematic issues of the initial data on the reliability of elements with small volumes of statistical information are presented. The reliability models use algorithms for transforming the functions of Boolean algebra into probabilistic functions [8].

\section{Probability model of structure}

The form of network structure determines the probability of its connectivity. To assess the reliability of the complex network systems, an approach that involves starting an analysis on the selection of individual components is used. The complex structure is decomposed into either successive or parallel subsystems, for each of which it is easy to estimate reliability [9]. The modeling of structural reliability relies on the notion of an operating condition in which all nodes of the structure are interconnected.

The system may have structural redundancy, which is formed by additional parallel connections between its elements in the form of rings. Accordingly, non-operating condition occurs with the loss of communication with any of the nodes of the structure. The probability of network structure connectivity is the probability of a sum of compatible events [3]:

$$
\begin{aligned}
R= & \sum_{i=1}^{T} P\left(B_{i}\right)-\sum_{i \neq j} P\left(B_{i} \cap B_{j}\right)+\ldots \\
& +(-1)^{T-1} P\left(B_{1} \cap B_{2} \cap \ldots \cap B_{T}\right)
\end{aligned}
$$

where $P\left(B_{i}\right)$ is the probability of forming a tree $B_{i}$, which connects all the nodes of the structure, $P\left(B_{i} \cap B_{j}\right)$ is the probability of simultaneous existence of spanning trees $B_{i}$ and $B_{j}$ in the structure of system.

Figure 1 shows a part of the city plan with a fragment of the reserved water supply network between its nodes $47,48,49,50$, which corresponds to the structure in Fig. 2. Let's assume that these nodes are designated respectively $v_{1}, v_{2}, v_{3}, v_{4}$.

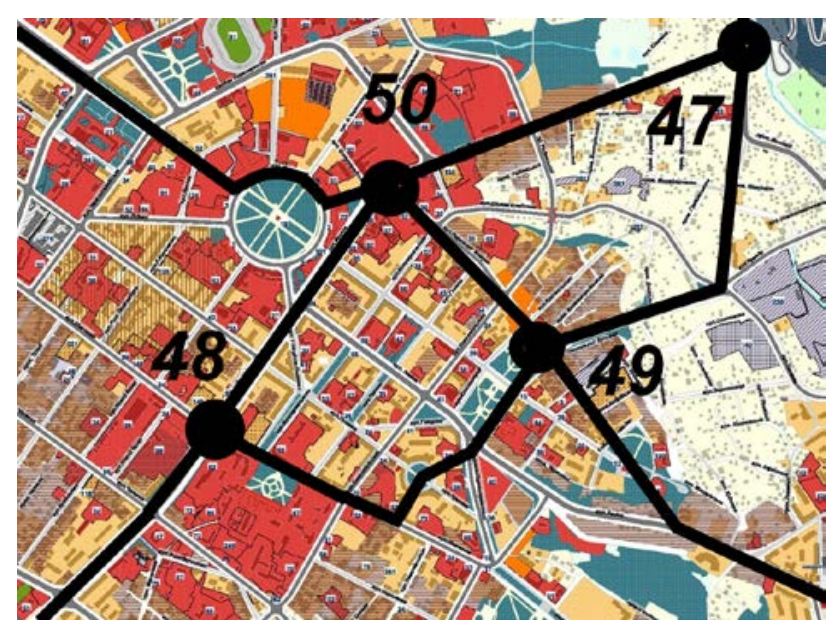

Figure 1. Fragment of the reserved water supply network

Consider the connectivity probability modeling of the redundant structure $S^{5}$, that has two rings $C_{1}^{3}, C_{2}^{3}$ with three sites in each ring $S^{5} \subset C_{1}^{3}, C_{2}^{3}$ (Fig. 2). One site is common for two rings of the structure: $d_{5} \in C_{1}^{3}, C_{2}^{3}$.

Let's assume that the sites of the structure have different reliability: $r_{1}^{d} \neq r_{2}^{d} \neq \ldots \neq r_{5}^{d}$. Limitation: the nodes' reliability is considered absolute: $r_{1}^{v}=r_{2}^{v}=\ldots=r_{4}^{v}=1$. The reliability value (probability of connectivity) varies within the range of rational numbers $(0,1)$. 


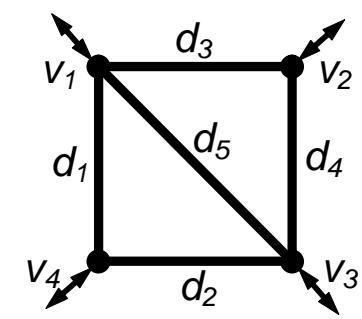

Figure 2. Double-ring redundant structure with 4 nodes and 5 sites

The redundant structure from five sites $d_{1}, \ldots, d_{5}$ connects four nodes $v_{1}, \ldots, v_{4}$ (Fig. 2). All possible unique operating conditions of the redundant structure from 5 sites will be $2^{5}-1=31$.

The number of unique operating conditions of the structure equals 14 (Fig. 3). Solid lines in the figure indicate operating connections of the structure whereas dashed lines stand for non-operating.

Analytically, the connectivity probability of the $S^{5}$ structure is expressed by a one-parameter polynomial:

$$
R=4 r^{5}-11 r^{4}+8 r^{3}
$$

where $r$ is reliability of the sites of the structure, $r \in(0,1), R \in(0,1)$. This expression is expedient to apply when the reliability of all units are significantly lower than the maximum value [10]: $r<<1$ or $q>>0, q \in(0,1), R \in(0,1)$.

From the other point of view, if the reliability of the sections of the network is much larger than the minimum value ( $q<<1$ or $r>>0$ ), then the other method is used, in which instead of the variable $r$ equation (2) is applied the difference $1-q$ :

$$
R=-4 q^{5}+9 q^{4}-4 q^{3}-2 q^{2}+1
$$

where $q$ are the insecure parts of the structure in Fig. 2, $q \in(0,1), R \in(0,1)$.

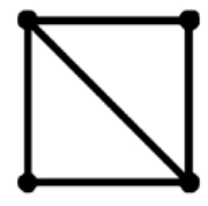

(a)

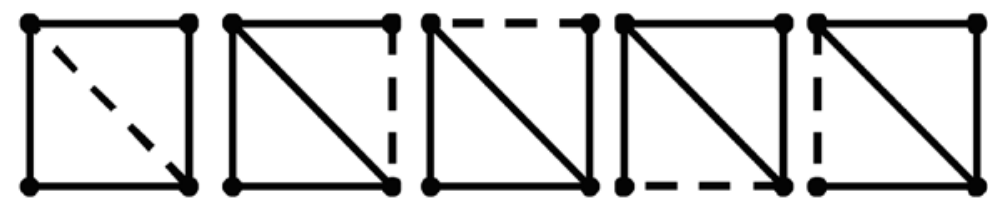

(b)

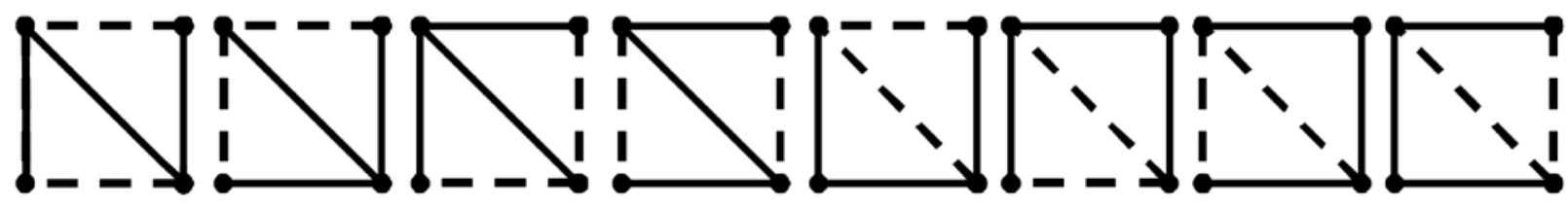

(c)

Figure 3. Fourteen unique operating conditions of the redundant structure of five sites: (a) all sites are operating; (b) failure of one site; (c) failure of two sites

Due to the redundancy of the structure, one does not exist, but several different connections between the nodes. Reliability of the redundant structure of five sites $d_{1}, \ldots, d_{5}$ of different reliability $r_{i} \neq r_{j}, i, j$ $=1, \ldots, 5$ and four nodes $v_{1}, \ldots, v_{4}$ is defined using logical probabilistic methods [8] and the graph theory [10]. It is described by a multi-parameter function [11]. The well-known formulas are rather cumbersome; therefore, a more convenient and compact form of the general expression of structural reliability for double-ring structures is proposed. The equation of the probability of the connectivity of the structure is introduced;

$$
\left.R=M\left(1+e_{p}+\sum_{i=1}^{p-1} e_{i}\left(1+e_{p}\right)+\sum_{i=1}^{j} e_{i} \sum_{i=j+1}^{p-1} e_{i}\right)\right)
$$

where $e_{i}=\frac{1-r_{i}}{r_{i}}, \quad M=\prod_{i=1}^{p} r_{i}, i=1,2, \ldots, p, \quad r_{i}$ is reliability value of $i$-site of structure $S^{p}, p$-is the number of sites in the structure, $j$ - is the number of sites in the first cycle $C_{1}^{l}$. In particular, the expression of reliability for double-ring structures of five sections and four nodes (Fig. 2) equals: 


$$
R=M\left(1+e_{5}+\sum_{i=1}^{4} e_{i}\left(1+e_{5}\right)+\sum_{i=1}^{2} e_{i} \sum_{i=3}^{4} e_{i}\right)
$$

where $\quad e_{i}=\frac{1-r_{i}}{r_{i}}, M=\prod_{i=1}^{n} r_{i}, i=1,2, \ldots, n, \mathrm{r}_{\mathrm{i}}$ is site reliability, $i=1,2, \ldots, 5$.

\section{Geometric dependency model}

Computer methods, programs, systems and tools of geometric modeling are extremely useful in a number of academic and industrial applications. Computer geometric modeling (CAGM) plays a significant role in the construction, creation and production of various objects [6]. The geometric model of an object is used to better understand its structure or behavior. The model must represent geometric, topological, and other specific data [12]. In the study of the connections between the variable values of various complex systems, functions are investigated, which are influenced by a certain number of factors. These connections are represented by geometric models of objects of multidimensional space - varieties. In formation and investigation of multidimensional geometric objects the point set is used. The latter is considered to be zero-dimensional varieties, which kinematically form objects of higher dimensionality. By means of moving in one direction in the space of a onedimensional object (line), which in the general case changes its shape and dimensions, a 2-variety (surface) of a certain area as one of its metric properties is formed. It is represented by its framework of lines. The latter may have several representations, for example, containing exclusively directrixes or generating lines, or both of its constituents. Moving in the same direction, in space surface forms a three-dimensional object, that is, the body of a certain volume, which is a onedimensional set of surfaces. Then, if we use the 3rd dimension of the received object, the number of frame types increases significantly. For generalization of the above principles of formulation apply to the representation of geometric objects of higher dimensionality. From the standpoint of geometry equation (7) is a multidimensional figure $B^{5}$ in the coordinate system $0 R, r_{1}, r_{2}, r_{3}, r_{4}, r_{5}$, where $R$ is a function, and the remaining variables are arguments. The variety $B^{5}$ is set in space $\Pi^{n}$, where $R-$ is a function dependent on the other five variables. In an analytical representation this object of multidimensional space describes 5 dependencies. Their visual representation in spaces of various dimensions, including two-dimensional (on a projective plane) or three-dimensional for solving problems of prediction and optimization of structural reliability is quite complicated. Therefore, we consider the possibilities of graphic modeling of this dependence.

Multidimensional object $B^{5}$ of the space $\Pi^{n}$ is formed by other geometric shapes $B^{1}, B^{2}, B^{3}, B^{4}$ of smaller dimensionality. When entering another variable value $r_{2}$ into the equation of onedimensional object (line) $B^{1}$, then from the standpoint of geometry, the arranged set of lines forms an object $B^{2}$ of greater dimension, which is a surface:

$$
\begin{aligned}
& B_{i}^{2}\left(r_{1}, r_{2}\right)=f\left(B_{i}^{1}\left(r_{1}\right), r_{2}\right) \\
& i=1,2, r_{3}, r_{4}, r_{5}=\text { const, }
\end{aligned}
$$

where $B_{i}^{1}\left(r_{1}\right)$ - are expressions of a onedimensional object $B^{1}$ (line). Accordingly, variety $B^{5}$ is formed by one-dimensional set of objects $B^{4}$.

$$
\begin{gathered}
R=M\left(1+c_{5}+\sum_{i=1}^{4} e_{i}\left(1+c_{5}\right)+\sum_{i=1}^{2} e_{i} \sum_{i=3}^{4} e_{i}\right) \\
, i=1,2,3,4 \\
r_{5}=\text { const }, c_{5}=\text { const. }
\end{gathered}
$$

For example, with the values $r_{3}=r_{4}=r_{5}=0.95$ hyperbolic paraboloid surface (Fig. 4) is formed:

$$
R=0.9975 r_{1} r_{2}+1.0379\left(1-r_{1}\right) r_{2}+0.9025 r_{1}\left(1-r_{2}\right)
$$

having a plane parallelism $0 R r_{2}$ and directrixes

$$
\begin{gathered}
R=0.9025\left(1-r_{2}\right) \\
R=1.0379 r_{2}
\end{gathered}
$$

In a more general form, the analytical expression of a geometric object $B^{2}$ :

$$
R=a r_{1} r_{2}+b\left(1-r_{1}\right) r_{2}+c r_{1}\left(1-r_{2}\right)
$$

and its directrixes $R=b r_{2}, R=c\left(1-r_{2}\right)$. 


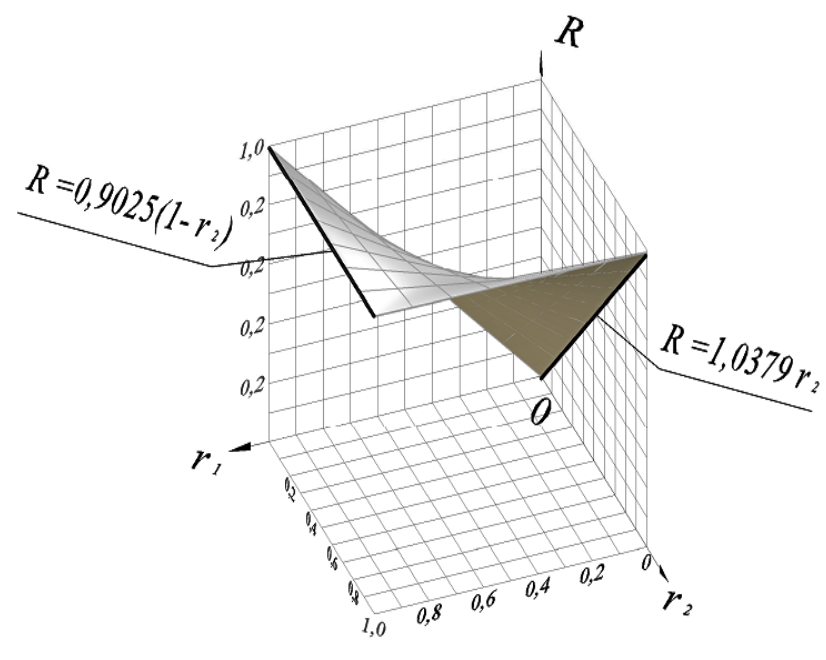

Figure 4. Image of equation (7) in the form of a twodimensional object - hyperbolic paraboloid with directrixes, $r_{3}, r_{4}, r_{5}=0.95$

Equation (10) is included in (5), which has even higher dimensionality. Accordingly, onedimensional set of two-dimensional objects $B^{2}$ (surfaces) forms a three-dimensional geometric object $B^{3}$ (body).

$$
\begin{gathered}
B_{i}^{3}\left(r_{1}, r_{2}, r_{3}\right)=f\left(B_{i}^{2}\left(r_{1}, r_{2}\right), r_{3}\right) \\
i=1,2,3, r_{4}, r_{5}=\text { const }
\end{gathered}
$$

where $B_{i}^{2}\left(r_{1}, r_{2}\right)$ - are expressions (8) of a twodimensional object $B^{2}$.

Figure 5 shows five surfaces with different curvature, that correspond values $r_{3}=r_{4}=r_{5}=0.95$, $0.75,0.58,0.35,0.15$.

Frame of the surface of the lines $R=a r_{1}+b$ is shown on the Fig. 6. A linear representation of the structural reliability of the system in this case is obtained under condition $r_{3}, r_{4}, r_{5}=$ const and $r_{2}=0$, $0.025, \ldots, 0.999$.

The coefficients of the equation of the lines $B^{1}$, forming an object $B^{5}$, are calculated as a result of the assignment of variables $r_{2}, r_{3}, r_{4}, r_{5}$ the corresponding values.

Setting of values for variables in multiparameter dependence corresponds to the operations of the introduction of plane levels, parallel to the corresponding coordinate fields. These planes form sections of the object of study, reducing its dimensionality to certain levels. Figure 5 shows the surface $B^{2}$ - a two-dimensional section of the object $B^{5}$. This surface is cut by a plurality of planes to produce objects of smaller dimension - lines.

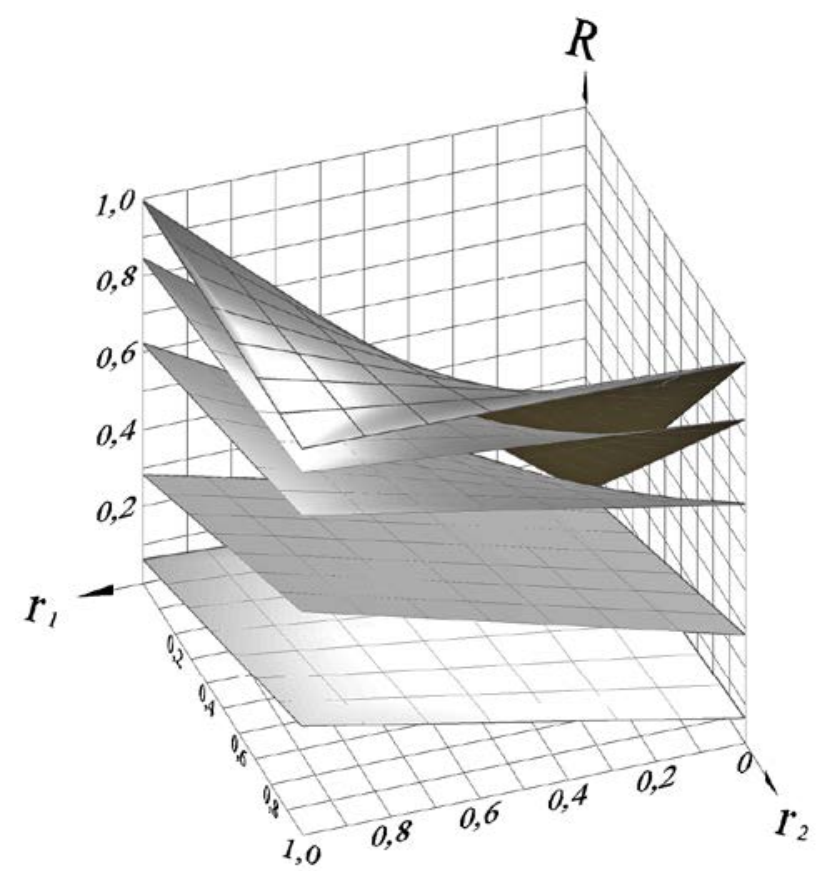

Figure 5.Parallel projection of three-dimensional sections of a three-dimensional object with a design vector $-1,-1,-1$

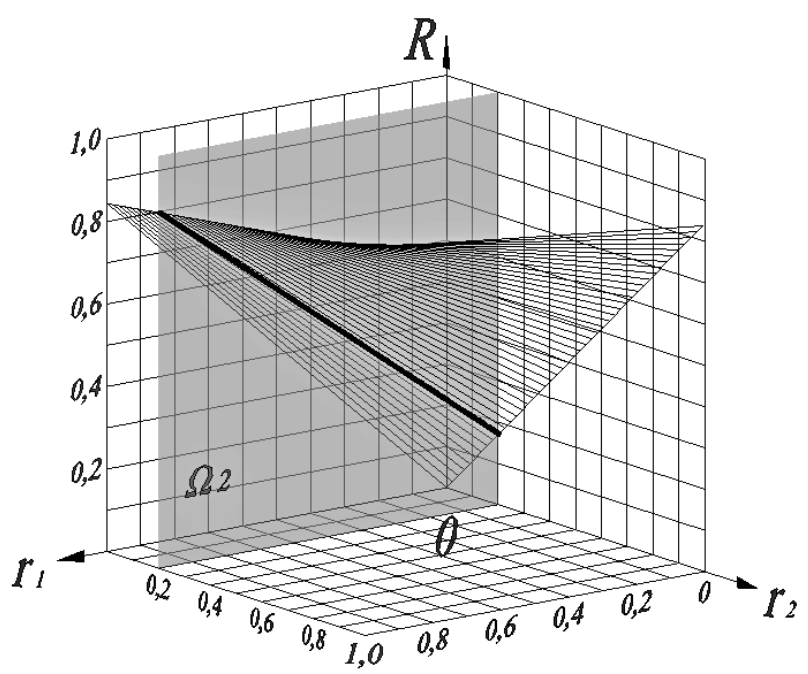

Figure 6. Frame of the surface of the lines $R=a r_{1}+b$

For example, cutting with one plane $\Omega_{2}$ which is parallel to the coordinate field $0 R_{1}\left(r_{2}=0.2\right)$ forms a straight line $R=0.714 r_{1}+0.208$.

The next cutting with a plane which is parallel to $O R r_{2}$ forms a geometric object of zero dimension - a point with coordinates $r_{1}, r_{2}, r_{3}, r_{4}, r_{5}$ in a multidimensional space that simulates the 
instantaneous state of reliability of the investigated system.

Thus, the geometric model of reliability is a structured variety. The tasks of forecasting and optimization of reliability [13], [14], [15], [16] are solved by reducing the dimension of the structured variety using the "cutting system."

\section{Conclusion}

Expressions of the connectivity probability of a structure having two cycles and five sites are represented as functions of one, as well as many variables. The compact form of accurate, but cumbersome expression of reliability of the doublering structure is convenient for computer modeling. The multiparametric dependence is modeled by a multidimensional geometric object, which allows us to analyze the connectivity probability of structures. The geometric model of the connectivity probability of the structure considers the different reliability of the elements. The use of computer graphics allows increasing demonstrativeness of modeling the connectivity probability of structures and significantly expands the capabilities of prediction and optimization of the reliability of the research object.

\section{Acknowledgement}

The authors are obliged to the Poltava National Technical Yuri Kondratyuk University for the support.

\section{References}

[1] Ozger, S., Mays, L. W.: Optimal Location of Isolation Valves in Water Distribution Systems: a Reliability / Optimization Approach 7.1-7.27. http://www.public.asu.edu/ lwmays/Ch07_Ma ys_144381-9.pdf

[2] Swamee, P. K., Sharma, A. K.: Design of Water Supply Pipe Networks, WileyInterscience, Hoboken, New Jersey, 2008.

[3] Reinschke, K., Ushakov, I.: Application of Graph Theory for Reliability Analysis, Verlag Technik, Berlin, 1987.

[4] Quang, H. T., Hara, Y.: Risks and performance in supply chain: the push effect, International Journal of Production Research, 2017.

[5] Scheibe, K. P., Blackhurst, J.: Supply chain disruption propagation: a systemic risk and norma accident theory perspective,
International Journal of Production Research, 2017.

[6] Geometric Modeling: Techniques, Applications, Systems and Tools, edited by Muhammad Sarfraz, Springer, Dordrecht, Boston, (2004), 3-5.

[7] Ryabinin, I. A.: Reliability of Engineering Systems. Principles and Analysis, MIR Publishers, Moscow, 1976.

[8] Ryabinin, I. A.: Logical-Probabilistic Calculus: A Tool for Studying the Reliability and Safety of Structurally Complex Systems, Automation and Remote Control, 64, 11771185 (2003).

[9] Tung, Y. K.: Evaluation of water distribution network reliability, Proceedings of the Specialty Conference ASCE, (1985), 1, 1-6.

[10] Reinschke, K. J.: Multivariable Control, A Graph Theoretic Approach, Springer Verlag, Berlin, Heidelberg, 1988.

[11] Reinschke, K., Ušakov, I. A.: Zuverlässigkeitsstrukturen. Modellbildung Modellauswertung. (Reliability structures. Modeling - Model Evaluation). In ZAMM Journal of Applied Mathematics and Mechanics, (1987), 312, (in German)

[12] Wolter, F. E., Reuter, M., Peinecke, N.: Geometric Modeling for Engineering Applications, In Encyclopedia of Computational Mechanics (eds E. Stein, R. Borst and T.J.R. Hughes), 2007, 5-8.

[13]Usenko, V.G., Vorontsov, O.V.: General approach to the problem of technical control systems, European Applied Sciences, ORT Publishing, Stuttgart, 2 (2013), 5, 40-42.

[14] Usenko, V., Kodak, O., Usenko, I.: Approximational projection of a network with an arbitrary structure, International Journal of Engineering \& Technology, 7 (3.2), (2018), 550-554.

[15] Usenko, V., Kodak, O.: Probability of the connection of the double structures of engineering networks with various reliability of parts, Academic Journal. Series: Industrial Machine Building, Civil Engineering, 1 (50), (2018), 270-277.

[16] Usenko, V., Pogorily, D., Usenko, I.: Problematic issues of methodology for ensuring the reliability of engineering networks, Applied Mechanics Reviews, Issue 6 (2), Volume 70, (2018), 1138-1146. 